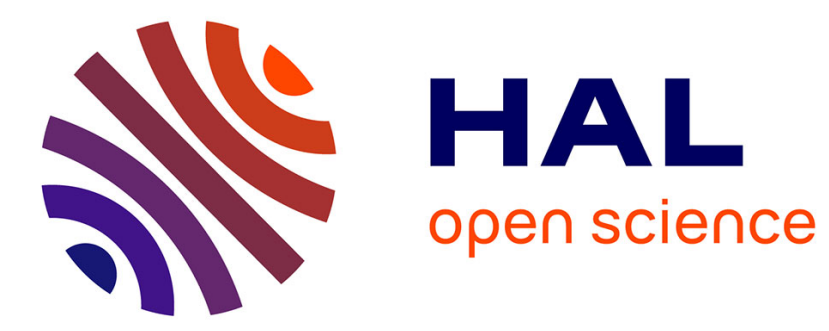

\title{
Adsorption isotherms for dilute solutions via the Mean Force method
}

\author{
Werner Billes, Rupert Tscheliessnig, Lukas Sobczak, Martin Wendland, \\ Johann Fischer, Jiří Kolafa
}

\section{- To cite this version:}

Werner Billes, Rupert Tscheliessnig, Lukas Sobczak, Martin Wendland, Johann Fischer, et al.. Adsorption isotherms for dilute solutions via the Mean Force method. Molecular Simulation, 2007, 33 (08), pp.655-666. 10.1080/08927020701313745 . hal-00515011

\section{HAL Id: hal-00515011 \\ https://hal.science/hal-00515011}

Submitted on 4 Sep 2010

HAL is a multi-disciplinary open access archive for the deposit and dissemination of scientific research documents, whether they are published or not. The documents may come from teaching and research institutions in France or abroad, or from public or private research centers.
L'archive ouverte pluridisciplinaire HAL, est destinée au dépôt et à la diffusion de documents scientifiques de niveau recherche, publiés ou non, émanant des établissements d'enseignement et de recherche français ou étrangers, des laboratoires publics ou privés. 


\section{Molecular Simulation \\ Journal of \\ Experimental Nanoscience

\section{Adsorption isotherms for dilute solutions via the Mean Force method}

\begin{tabular}{|r|l|}
\hline Journal: & Molecular Simulation/Journal of Experimental Nanoscience \\
\hline Manuscript ID: & GMOS-2007-0004 \\
\hline Journal: & Molecular Simulation \\
\hline Date Submitted by the \\
Author: & 08-Jan-2007 \\
\hline Complete List of Authors: & $\begin{array}{l}\text { Billes, Werner; BOKU - University of Natural Resources and Applied } \\
\text { Life Sciences, Institute for Chemical and Energy Engineering } \\
\text { Tscheliessnig, Rupert; BOKU - University of Natural Resources and } \\
\text { Applied Life Sciences, Institute for Chemical and Energy } \\
\text { Engineering } \\
\text { Sobczak, Lukas; BOKU - University of Natural Resources and } \\
\text { Applied Life Sciences, Institute for Chemical and Energy } \\
\text { Engineering } \\
\text { Wendland, Martin; BOKU - University of Natural Resources and } \\
\text { Applied Life Sciences, Institute for Chemical and Energy } \\
\text { Engineering } \\
\text { Fischer, Johann; BOKU - University of Natural Resources and } \\
\text { Applied Life Sciences, Institute for Chemical and Energy } \\
\text { Engineering } \\
\text { Kolafa, Jiří; Institute of Chemical Technology, Department of } \\
\text { Physical Chemistry }\end{array}$ \\
\hline Keywords: \\
\hline $\begin{array}{l}\text { Adsorption Isotherm, Dilute Solution, Local Density, Mean Force } \\
\text { Method }\end{array}$ \\
\hline \hline
\end{tabular}

\section{SCHOLARONE Manuscripts}




\title{
Adsorption isotherms for dilute solutions via the Mean Force method
}

\author{
W. BILLES $\dagger$, R. TSCHELIESSNIG $\dagger$, L. SOBCZAK $\dagger$, M. WENDLAND $\dagger$, J. FISCHER $* \dagger$, \\ and J. KOLAFA \\ $\dagger$ Institute for Chemical and Energy Engineering, BOKU-University of Natural Resources \\ and Applied Life Sciences, A-1190 Vienna, Austria
}

$\ddagger$ Department of Physical Chemistry, Institute of Chemical Technology, CZ-166 28 Prague 6, Czech Republic

\footnotetext{
*Author for correspondence: Johann Fischer, Institut für Verfahrens- und Energietechnik, Universität für Bodenkultur, Muthgasse 107, A-1190 Wien, Tel.: +43 13709726 201, Fax: +43 13709726 210, email: johann.fischer@boku.ac.at
} 
Postal addresses, telephone and fax numbers, and email addresses of the authors:

Werner Billes: Muthgasse 107, A-1190 Vienna, Tel: +43 13709726 203, Fax: +43 1 3709726 210, email: werner.billes@ boku.ac.at

Rupert Tscheliessnig: Muthgasse 107, A-1190 Vienna, Tel: +43 13709726 202, Fax: +4313709726 210, email: rupert.tscheliessnig@boku.ac.at

Lukas Sobczak: Muthgasse 107, A-1190 Vienna, Tel: +43 13709726 200, Fax: +43 1 3709726 210, email: lukas_sobczak@hotmail.com

Martin Wendland : Muthgasse 107, A-1190 Vienna, Tel: +43 13709726 212, Fax: +43 1 3709726 210, email: martin.wendland@boku.ac.at

Johann Fischer : Muthgasse 107, A-1190 Vienna, Tel: +43 13709726 201, Fax: +43 1 3709726 210, email: johann.fischer@boku.ac.at

Jiří Kolafa: Technická 5, CZ-166 28 Prague 6, Tel: +420 22435 4257, Fax: +420 22431 0273, email : jiri.kolafa@vscht.cz 
The adsorption of solutes from a dilute liquid solution is of great technical importance but calculations of the local density of the solute and of the adsorption isotherm by standard molecular simulation yield large scattering with increasing dilution. As alternative the mean force $(\mathrm{MF})$ method was suggested where the mean force on a constrained solute molecule is integrated over a path from the bulk fluid to the wall. It has already been shown that the MF method gives reliable results for the relative local density, even at high dilution. Here, an extension of this method is introduced, where the absolute value of the bulk density is determined by particle balance. Thus, it is possible to calculate adsorption isotherms from the Henry regime to any finite concentration. Molecular dynamics simulations for the local density and the adsorption isotherm were performed for a model solution consisting of tetrahedral Lennard-Jones (LJ) solvent and linear LJ solute molecules in contact with a plane wall. It is found that the MF-results show less scattering than the results from standard simulations. Moreover, results for the orientation and the selectivity are given.

Keywords: Adsorption Isotherm; Dilute Solution; Local Density; Mean Force Method 


\section{Introduction}

In many engineering and biological applications we encounter adsorption from dilute liquid solutions. Examples are recovery of proteins via chromatography, adsorptive removal of organic impurities from water or adsorption of pesticides from soil material. In order to understand the structure of the adsorbate and to predict adsorption isotherms molecular simulations can be helpful.

The problem with standard molecular simulations, however, is in the fact that in case of low solute concentrations it might need rather long time for the solute molecules to move to the wall and their local density profiles might be subject of large statistical uncertainties, as was shown earlier [1-3]. Even for solutions of spherical molecules the local densities from standard simulations showed strong scattering [1]. In order to overcome that problem we have suggested using the mean force (MF) method [1-3]. In that method, one solute particle $\mathrm{B}$ is kept with one reference site at a fixed position and the MF exerted on that site from the other particles and from the wall is calculated. Next, the reference site is moved to a position closer to the wall and again the MF is calculated. Continuing, the reference site is moved on a path towards the wall and the MF values are recorded. Then, by integration of the MF along that path, the change of the potential of mean force (PMF) is obtained which is the change of the free energy along that path. Therefrom the ratio of the local density of the solute at the end point divided by the local density at the starting point of the path can be calculated. This approach is valid for any type of molecules and for any solute concentration and has been applied already to solutions of spherical Lennard-Jones (LJ) 
molecules [1], to solutions of linear in spherical molecules [2], and to the solution of benzene in water [3].

So far, the MF method works well also for dilute solutions but the problem hitherto was the determination of the absolute local density which is required for the calculation of the adsorption isotherm. In order to overcome this problem we suggest in this paper to use the particle balance equation for the determination of the absolute local density of the solute.

In addition to the presentation of the theory the purpose of this paper is to explore the feasibility of the MF method in combination with the particle balance equation for the calculation of the adsorption isotherm for a dilute mixture in case of a 'simple system'. The latter means not too strong adsorption and molecules which do not demix in the bulk liquid. We consider here a model consisting of tetrahedral LJ solvent and linear LJ solute molecules in contact with a plane LJ 9/3 wall which is in line with earlier investigations [1, 2]. In order to ensure a 'simple system' the model parameters were selected to mimic the adsorption of ethane from a dilute liquid solution with methane in slit pores of graphite at the temperature $T=160 \mathrm{~K}$. Application of the method to adsorption of more complex systems as e.g. benzene from water on graphite [3], which shows very strong adsorption and liquid phase separation, will be considered in a subsequent study. The molecular simulation method to be used here is molecular dynamics (MD) and the particular code is MACSIMUS (MACromolecular SIMUlation Software) [4] as previously [1-3].

The literature on molecular simulations of adsorption has become rather extensive and hence we concentrate here on some papers relevant for the present work. For mixtures of spherical LJ molecules an extensive comparison of results from density functional theory with MD results was made in [5] including gas-liquid transitions. There are also some 
papers on the adsorption of mixtures of methane + ethane. In [6] GCMC simulations for adsorption from the gas phase in a slit pore were reported and the selectivity was studied as function of the pressure. The same method was used in [7] in order to study adsorption in MCM-41 for pressures up to 33 bar and temperatures between $265 \mathrm{~K}$ and $373 \mathrm{~K}$. GCMC simulations were also performed for adsorption of the mixed gas in heterogeneous materials at 298.15 K [8]. Finally a MC/GCMC study on vapor-liquid equilibria of methane + ethane adsorbed in a slit mesopore has to be mentioned [9]. In this paper, the mole fraction of ethane in the bulk gas phase at $126 \mathrm{~K}$ was 0.05 and the amount of methane and ethane inside the pore was calculated as function of the pressure.

The last mentioned paper [9] is the one of closest relevance to the present work. We will, however, explore concentrations of the solute with mole fractions in the bulk liquid below 0.05 down to infinite dilution. Moreover, we want to emphasise that our main aim is to compare the performance of the suggested new method with standard simulations, whilst we will not focus on comparison with experimental data for two reasons. First, it was already shown by Yun et al. [7] that the LJ model for methane and the two-centre LJ model for ethane suggested by us earlier [10] yield excellent agreement of simulations with adsorption experiments. We expect that the present tetrahedral model might be no better description for methane but it is more challenging for exploring the method. Second, we are not aware of experimental data for adsorption of ethane from a solution with methane at such low concentrations. The development of simulation methodologies for these low concentrations, however, is an interesting challenge as e.g. the mole fraction for the solubility of benzene in water is at room temperature only $4.4 \times 10^{-4}$ and is lower by two orders of magnitude for some hormones in water. 
In the next section the theory will be presented. Thereafter, the model of the liquid mixture at the graphite wall and the simulation methodology will be specified. Then, results from standard MD simulations and from the MF method in combination with the particle balance will be given for the local densities and the adsorption isotherm for low solute concentrations. Moreover, results for the orientation and the selectivity will also be given.

\section{Theory}

The derivation of the MF method in order to obtain the relative local density of a solute in case of adsorption was given by Billes et al. [1]. For better understanding and further development of the approach to determine adsorption isotherms, key formulas and short explanations are given in the following.

We consider a fluid mixture with solvent A-particles and solute B-particles in contact with a wall. In the MF-method, one solute particle B is kept with one reference site at a fixed position $\boldsymbol{r}$ and the $\mathrm{MF}<\boldsymbol{F}_{B}(\boldsymbol{r})>$ exerted on that site from the other particles and from the wall is calculated. Next, the reference site is moved to a position closer to the wall and again the MF is calculated. Continuing, the reference site is moved on a path and the MF values are recorded. Then, by integration over the MF along that path denoted by $s$, the change of the potential of mean force $(\mathrm{PMF}) \Delta w$ is obtained which is also the change of the Helmholtz free energy $\Delta A$

$$
\Delta w=\Delta A=-\int<\boldsymbol{F}_{B}(\boldsymbol{s})>\cdot d \boldsymbol{s}
$$


where the change $\Delta$ refers to the difference between the end point $\boldsymbol{r}_{2}$ and the starting point $\boldsymbol{r}_{1}$ of the path $s$. Next, it is known that the local density $n_{B}(\boldsymbol{r})$ is related to the change of the PMF according to

$$
\Delta w=-k T \Delta \ln n_{B}(\boldsymbol{r})
$$

or

$$
n_{B}\left(\boldsymbol{r}_{2}\right) / n_{B}\left(\boldsymbol{r}_{1}\right)=\exp \{-\beta \Delta w\}
$$

with $\Delta w$ given by Eq. (1) and $\beta=1 / k T$. This approach is valid for any type of molecules and for any solute concentrations and has been applied so far to solutions of spherical Lennard-Jones molecules [1], to solutions of linear in spherical molecules [2], and to the solution of benzene in water [3].

The new point to be addressed here is the absolute value of the local density which can be obtained via a particle balance. Trivially, integrating the local density over the whole fluid space must yield the total number $N_{B}$ of solute particles

$$
\int n_{B}\left(\boldsymbol{r}_{2}\right) d \boldsymbol{r}_{2}=N_{B}
$$

Then, by inserting Eq. (3) into Eq. (4) one obtains

$$
n_{B}\left(\boldsymbol{r}_{1}\right) \int \exp \{-\beta \Delta w\} d \boldsymbol{r}_{2}=N_{B}
$$

and finally, inserting Eq. (5) into Eq. (3) yields

$$
n_{B}\left(\boldsymbol{r}_{2}\right)=N_{B} \exp \{-\beta \Delta w\} / \int \exp \{-\beta \Delta w\} d \boldsymbol{r}_{2}
$$

Note that the integration $\int \exp \{-\beta \Delta w\} d \boldsymbol{r}_{2}$ has to be done over the whole volume. In case that the starting point $\boldsymbol{r}_{l}$ is in the bulk fluid, $n_{B}\left(\boldsymbol{r}_{1}\right)$ should be the bulk density $n_{B b}$ and hence

$$
n_{B b}=N_{B} / \int \exp \{-\beta \Delta w\} d \boldsymbol{r}_{2}
$$


Our particular interest here is to study adsorption on a planar wall. For technical reasons it is convenient to study the situation in a sufficiently wide slit pore of width $L$ where the fluid in the middle can be considered as bulk fluid. In this case it is appropriate to start in the middle plane of the pore, to consider a path $s$ perpendicular to the walls, and to denote the distance from the one wall by $z$. Then Eq. (7) simplifies to

$$
\begin{gathered}
L \\
n_{B b}=N_{B} /\left(A \int \exp \{-\beta \Delta w\} d z\right),
\end{gathered}
$$

0

where $A$ is the surface of the simulation box parallel to the walls. Moreover, for symmetry reasons we have

$$
\exp \{-\beta \Delta w(z)\}=\exp \{-\beta \Delta w(L-z)\}
$$

which yields by insertion into (8)

$$
\begin{gathered}
L / 2 \\
n_{B b}=N_{B} /\left(2 A \int \exp \{-\beta \Delta w\} d z\right), \\
0
\end{gathered}
$$

and finally we get the density profile as

$$
n_{B}(z)=n_{B b} \exp \{-\beta \Delta w\}
$$

Moreover, we still want to express the adsorption excess $\Gamma$ and the Henry constant $H$. The adsorption excess $\Gamma$ per unit area is defined as usually as

$$
\Gamma=(1 / A) \int\left[n_{B}(\boldsymbol{r})-n_{B b}\right] d \boldsymbol{r}
$$

or in case of planar geometry 


$$
\Gamma=\int\left[n_{B}(z)-n_{B b}\right] d z
$$

The Henry constant $H$ is defined as

$$
\Gamma=H n_{B b}
$$

in the limit of low $n_{B b}$. By using Eq. (12) this yields

$$
H=(1 / A) \int\left[n_{B}(\boldsymbol{r}) / n_{B b}-1\right] d \boldsymbol{r}
$$

and by using Eq. (6) and (7) one obtains

$$
H=(1 / A) \int[\exp \{-\beta \Delta w\}-1] d \boldsymbol{r},
$$

and in case of planar geometry

$$
H=\int[\exp \{-\beta \Delta w\}-1] d z .
$$

Note that Eq. (12) to (17) hold for a semi-infinite fluid, and in case of a wide planar slit pore the integrations have to be performed from one wall to the middle plane.

Summarising, for the calculation of an adsorption isotherm for adsorption on a planar wall it is convenient to use a sufficiently wide slit pore. Starting from a point in the middle plane where a bulk state can be assumed, the MF in the $z$-direction $\left\langle F_{B}(z)\right\rangle$ has to be calculated for several points along a perpendicular path $s$ from the bulk to close to the surface. From the MF the PMF can be calculated using Eq. (1). Therefrom the bulk fluid density $n_{B b}$ can be calculated from Eq. (10) and the absolute local density $n_{B}(z)$ from Eq. (11). Then $n_{B}(z)$ is integrated to yield the surface excess $\Gamma$ from Eq. (13). Repeating the procedure for different numbers $N_{B}$ of solute particles yields the surface excess $\Gamma$ as function of the solute bulk density $n_{B b}, \Gamma=\Gamma\left(n_{B b}\right)$, which is just the adsorption isotherm. 
Finally, for further quantification of the adsorption process usually the selectivity $S_{B A}$ is introduced as the ratio of the mole fractions in the adsorbed phase $x_{B} / x_{A}$ to the ratio of the mole fractions in the bulk $y_{B} / y_{A}[11]$

$$
S_{B A}=\left(x_{B} / x_{A}\right) /\left(y_{B} / y_{A}\right) .
$$

In case of fluids in pores this definition can be directly used [7, 8], in case of adsorption on a plane wall the definition has to be somewhat modified. For multilayer-adsorption from the gas phase, the ratio $x_{B} / x_{A}$ can be well approximated by the ratio of the surface excess quantities $\Gamma_{B} / \Gamma_{A}[12]$; note that the surface excess defined in Eq. (12) is $\Gamma_{B}$ and similarly $\Gamma_{A}$ can be introduced. For multilayer-adsorption from the liquid the situation is more subtle and we decided to take the concentrations in the first adsorbed layer as measure for the concentrations in the adsorbed phase. Let us consider a plane wall and assume that the first peak of the local density of B-particles $n_{B}(z)$ starts at $z_{0 B}$ and ends at $z_{1 B}$, then the number of B-particles in the first adsorbed layer $N_{B 1}$ is given by

$$
\begin{gathered}
z_{1 B} \\
N_{B 1}=A \int_{n_{B}}(z) d z, \\
z_{O B}
\end{gathered}
$$

and an analogous expression can be given for the number of A-particles in the first layer $N_{A l}$. Therefore, the concentration ratio in the adsorbed layers can be expressed as

$$
x_{B} / x_{A}=N_{B 1} / N_{A 1}
$$

which then allows the calculation of the selectivity according to Eq. (18). 


\section{Model and Simulation Methodology}

Solutions of tetrahedral Lennard-Jones (LJ) solvent and linear LJ solute molecules in contact with a plane LJ 9/3 wall were investigated with standard simulations and with the above described MF method implemented into the MD simulation code MACSIMUS [4]. The intermolecular interactions were chosen to mimic the adsorption of ethane from a dilute liquid solution with methane in slit pores of graphite and were taken from the CHARMM21 force field [13] which is already implemented in MACSIMUS.

In the model, all site-site interactions are assumed to be 12/6 Lennard-Jones (LJ) potentials. The interaction parameters $\varepsilon_{\alpha \alpha}$ and $\sigma_{\alpha \alpha}$ between like interaction sites $\alpha$ and the bond lengths $l$ are given in Table 1 . The $\mathrm{CH}_{4}$ molecule is modelled fully atomistic by five sites, one site representing $\mathrm{C}$ and the other four sites representing $\mathrm{H}$. The $\mathrm{C}_{2} \mathrm{H}_{6}$ molecule is modelled by two $\mathrm{CH}_{3}$ sites. The wall consists of carbon atoms. For all unlike interactions $\varepsilon_{\alpha \beta}$ and $\sigma_{\alpha \beta}$ between sites $\alpha$ and $\beta$ the Lorentz-Berthelot combining rules [14-16] are assumed. Partial charges are set to zero.

Any interaction between a wall-carbon atom $\mathrm{c}$ and a fluid interaction site $\mathrm{s}$ is characterised by a $12 / 6 \mathrm{LJ}$ potential with parameters $\varepsilon_{c s}$ and $\sigma_{c s}$ which are obtained from the like interaction parameters given in Table 1 using the Lorentz-Berthelot combining rules. In order to simplify the model, the wall is assumed to be planar which is achieved by smearing out the graphite-carbon atoms over the infinite half-space $z \leq 0$ where we assume a number density $\rho_{c}=110.3 \mathrm{~nm}^{-3}$ which is close to the value given by Steele [17]. This means, for a fixed distance $z \geq 0$ of a fluid interaction site s ( $\mathrm{C}$ and $\mathrm{H}$ for methane; $\mathrm{CH}_{3}$ for ethane), the $\mathrm{LJ}$ interactions with parameters $\varepsilon_{c s}$ and $\sigma_{c s}$ are averaged over all carbon atoms in the half 
space $z \leq 0$. The resulting potential $u_{w}$ between the planar carbon wall and any interaction site $\mathrm{s}$ is then obtained as

$$
u_{w}=(2 / 3) \pi \varepsilon_{c s} \rho_{c} \sigma_{c s}^{3}\left[(2 / 15)\left(\sigma_{c s} / z\right)^{9}-\left(\sigma_{c s} / z\right)^{3}\right]
$$

The density profiles calculated for methane and ethane are those of the centre of mass (CoM) which is the carbon-atom in case of methane and the centre between the two $\mathrm{CH}_{3}$ groups in case of ethane. Taking as molecular length the carbon-carbon site-site diameter of methane $\sigma=\sigma_{C-C ; C H 4}=0.3207 \mathrm{~nm}$, the local density of methane is given by $n_{A}{ }^{*}=n_{A} \sigma^{3}$ with $n_{A}$ being the particle number density of methane, similarly the local density of ethane is given by $n_{B}^{*}=n_{B} \sigma^{3}$, and the adsorption excess is given by $\Gamma^{*}=\Gamma \sigma^{2}$. Forces are presented in Nanonewton $(\mathrm{nN})$, energies in $\mathrm{kJ} / \mathrm{mol}$, and distances in Nanometer ( $\mathrm{nm})$. Selectivities are dimensionless by definition.

Whilst we are in essence interested in the situation at one graphite wall, the simulations were performed for technical simplicity in a slit pore of sufficiently large width $L$ as already mentioned. Simulations were performed using standard MD as well as the MF-MD approach. In order to have a clear nomenclature, we call the standard MD simulations in which all particles move freely 'unconstrained', and those which are used in the MF method with one particle fixed at its reference site 'constrained' simulations. Input data were the total number of particles $N=400$ and the ratio of the ethane to methane particles which varied according to $1: 399,2: 398,5: 395,10: 390,20: 380,30: 370$, and 40:360. Moreover the temperature was prescribed to be $160 \mathrm{~K}$ and the mass density of the liquid was assumed to be $320 \mathrm{~kg} / \mathrm{m}^{3}$ which is a value close to the saturated liquid density of methane at $160 \mathrm{~K}$ [18]. The simulation box was cubic and adjusted by the code according to the number of 
particles and the mass density which resulted in a box length $L$ of about $3.2 \mathrm{~nm}-$ note that this is also the width of the slit pore. The exact values of $\mathrm{L}$ for the different particle ratios are given in Table 2 .

Further simulation details are that 1 ) periodic boundary conditions were used in directions parallel to the LJ walls, 2) the cut-off radius for the LJ interactions (the only one interactions in our simulation model due to a non-charged wall) is $1 \mathrm{~nm}$, which is less than half of the box length, 3) the temperature was kept constant by the Berendsen friction thermostat, 4) the length of the integration time steps was $\sim 1.67 \mathrm{fs} ., 5$ ) the total number of production time steps for obtaining a density profile was 600,000 in both methods, and 6) the first 500 time steps of each simulation run were taken as equilibration period and were not considered in obtaining the results.

In the unconstrained simulations the local densities $n_{A}(z)$ and $n_{B}(z)$ were determined by dividing the pore width $L$ into 1,000 intervals of equal width and counting the number of methane or ethane particle-centres in these intervals after each time step. For each particle ratio two separate simulation runs over 300,000 time steps were performed with different initial positions of the ethane molecules as given in Table 2. It should be pointed out that the second run was not a continuation of the first one. What one expects from sufficiently long runs in a symmetric pore is a) that results from different runs agree and b) that the right part of the density profile should be symmetric to the left part. It will, however, be seen in the next Section that for the dilute component these expectations are not achieved accurately in the duration of our simulation runs. Hence, in order to arrive at some well defined procedure for the calculation of the density profile $n_{B}(z)$, of the solute bulk density $n_{B b}$, and of the adsorption excess $\Gamma$ from unconstrained simulations we compute density 
profiles as averages from both simulation runs and from both the left and right half of the pore, if not stated otherwise. The final bulk density $n_{B b}$ from the unconstrained simulations was determined as the average of the local density $n_{B}(z)$ in the interval from $z=1.5 \mathrm{~nm}$ to $z$ $=L / 2$.

In the calculations of the local density $n_{B}(z)$ of the dilute component via the MF method, the reference site on the ethane molecule was put into its CoM. This was then fixed during one simulation run at a given distance $z$ from the left wall whilst the molecule was allowed to rotate and the MF on the CoM was calculated. This procedure was repeated for 60 evenly distributed distances $z$ starting from $z=1.700 \mathrm{~nm}$ which is close to the centre of the simulation box down to $z=0.225 \mathrm{~nm}$ which is close to the wall with 10,000 simulation time steps for each distance. Then, the PMF was calculated by integration of the MFs along a path perpendicular to the wall according to Eq. (1) with the starting point being just in the centre of the pore, i.e. $z_{1}=L / 2$, and the PMF being zero for $L / 2$. In case of more ethane molecules, one of them is constrained whilst the others move freely. The initial positions of the freely moving ethane particles are also given in Table 2. Having obtained the PMF, the bulk density $n_{B b}$ is calculated via the particle balance in the form of Eq. (10), and the density profile $n_{B}(z)$ is calculated from Eq. (11).

\title{
4. Results and Discussion
}

\author{
4.1. Density Profiles and Adsorption Isotherms \\ Unconstrained and constrained simulations were performed for the overall ratios of \\ ethane to methane particles being 1:399, 2:398, 5:395, 10:390, 20:380, 30:370, and 40:360.
}


First we present in Fig. 1 local density results for 1 ethane and 399 methane particles from two separate unconstrained simulation runs over 300,000 time steps each with the initial ethane positions as given in Table 2. In Fig. 1 the left and the right half of the methane and ethane profiles have been averaged. Whilst for the solvent the results from both runs are identical, the solute profiles show remarkable differences to be discussed below.

Let us first consider the rather distinct profile for the solvent, i.e. for the 5-centre methane model from Fig.1 which is similar also for the other particle ratios considered. Close to the wall we observe a first peak at $0.31 \mathrm{~nm}$ with a shoulder towards the wall and a second peak at $0.39 \mathrm{~nm}$. In order to understand that result we first note that the potential minimum of the $\mathrm{C}_{\mathrm{CH} 4}$ atom with respect to the wall is at a distance $z=0.294 \mathrm{~nm}$ and that of the $\mathrm{H}_{\mathrm{CH} 4}$ is at $z$ $=0.258 \mathrm{~nm}$. More important, however, is the fact that the orientation of the tetrahedral methane with respect to the wall can be the pyramid orientation with one plane parallel and close to the wall and the inverse pyramid orientation with one plane parallel but remote from the wall [19]. A calculation of the potential energy between the wall and the CoM of the 5-centre methane molecule in these selected orientations showed the minima to be at $z=$ $0.280 \mathrm{~nm}$ for the pyramid orientation and at $z=0.405 \mathrm{~nm}$ for the inverse pyramid orientation. These values are close to the first and the second peak of the density profile, which indicates that the first peak corresponds to the pyramid orientation and the second peak to the inverse pyramid orientation. The shoulder in the density profile of $\mathrm{C}_{\mathrm{CH} 4}$ may be explained by the fact that this is the favorite position for the $\mathrm{H}_{\mathrm{CH} 4}$ atoms. For distances further remote from the wall we observe the usual layering of the methane molecules as it is well known for spherical molecules [20] with a relaxation towards a bulk state. We also 
note that the first peak of the local reduced methane density $n_{A} *$ has a value of 0.92 whilst the bulk density in the centre of the pore amounts to 0.34 which is a ratio of 2.7 .

For the density profiles of ethane from both runs we observe in Fig. 1 a first maximum at $0.35 \mathrm{~nm}$ and a second at around $0.73 \mathrm{~nm}$. The potential minimum of the ethane molecule with respect to the wall is for parallel orientation at a distance $z=0.322 \mathrm{~nm}$ and for perpendicular orientation at $z=0.451 \mathrm{~nm}$. This suggests that ethane is preferentially adsorbed in near-parallel orientation. Moreover, the ratio of local density at the first peak to the bulk density is considerably higher than for methane. Details concerning the orientation and the selectivity, however, will be discussed later. The crucial point with these results is the scattering of the local ethane density in particular in the centre of the pore where we expect to have reached nearly bulk density. Actually the local densities from the two runs differ in the centre of the pore by a factor of two. Interesting to note is that in run 1 the ethane molecule was started in the centre of the pore (see Table 2) and the profile in this case is higher near the wall and lower in the centre than in case 2 where the ethane molecule was inserted into the first layer at the wall. The value of $n_{B b}$ determined as described above is given in Table 3 but we should keep in mind its large uncertainty.

Next, we present in Fig. 2 the MF and PMF results for the solute in the 1:399 mixture from constrained simulations. The MF crosses the $z$-axis several times. Trivially, every turn of the MF from plus to minus or vice versa results in a maximum or minimum of the PMF. The result for the PMF shown here is also the basis for the calculation of the Henry constant according to Eq. (17) to be given later.

The results for the local density $n_{B}(z)$ of ethane obtained from the constrained and the unconstrained simulations for the ethane-methane ratio 1:399 as described in Sec. 3 are 
compared in Fig. 3. This figure also contains an inset for a clear presentation of the results close to the centre of the pore. We observe that the density profiles $n_{B}(z)$ obtained from both methods agree only qualitatively. The first peaks occur at about the same distance but their heights are different. More important, however, are the differences of the local densities in the centre of the pore where the results differ by more than a factor of three. Of course, the unconstrained results must be higher there in order to compensate for the lower first peak. Moreover looking on the region from 1.2 to $1.6 \mathrm{~nm}$ in the inset we note that the local density from the constrained simulations remains nearly constant there whilst the unconstrained results increase by nearly a factor of 2 . The final results from both methods given in Table 3 differ for $n_{B b}$ by a factor of 3.6 and for $\Gamma$ by a factor of 0.45 .

Now we proceed to higher particle ratios. Density profiles $n_{B}(z)$ of ethane from constrained and unconstrained simulations for the ethane to methane ratio 2:398 are shown also in Fig. 3, whilst results for the particle ratios 5:395 and 10:390 are shown in Fig. 4, and for the particle ratios 20:380 and 30:370 in Fig. 5. These results will be discussed below together with those from the particle ratios 1:399 and 40:360.

Finally more detailed profiles from the unconstrained simulations together with the standard profile from the constrained simulations are shown in Fig. 6 for the particle ratio 40:360 which is the upper end of the solute concentration considered here. The figure shows the density profiles from runs 1 and 2 over the whole width of the pore. From Table 2 it can be seen that in run 1 the solute particles were inserted symmetrically according to their expected distribution whilst in run 2 the solute particles were inserted at random. From Fig. 6 we see that run 2 yields a more symmetric result than run 1 . This finding and the solute profiles in Fig. 1 indicate that there is apparently no correlation between the initial 
configuration and the final result. It is also interesting that the results from the unconstrained run 2 are rather close to those from the constrained simulations.

We are now in the position to compare the density profiles $n_{B}(z)$ from the unconstrained and constrained runs as well as the bulk fluid densities $n_{B b}$ and the adsorption excess values $\Gamma$ for all particle ratios by looking on Fig. 3 to 6 and on Table 3. In order to have a guideline for the appraisal of the results we may assume that for this 'simple' system the values of $n_{B}(z), n_{B b}$ and $\Gamma$ should be in a first approximation linear functions of $N_{B}$.

First we observe that the heights of the first peaks in the density profiles from the unconstrained and constrained simulations agree in general reasonably well. If we calculate now the peak heights $H$ divided by $N_{B}$ we obtain in the series $N_{B}=1,2,5,10,20,30,40$ for the unconstrained results the $H / N_{B}$ values $9.2 \times 10^{-3}, 10.0 \times 10^{-3}, 9.7 \times 10^{-3}, 9.7 \times 10^{-3}, 10.1$ x $10^{-3}, 8.8 \times 10^{-3}$, and $8.6 \times 10^{-3}$, where the values for $N_{B}=1$ and $N_{B}=20$ deviate most from a linear correlation. For the constrained results the $H / N_{B}$ values are $10.8 \times 10^{-3}, 10.6 \times 10^{-3}, 9.3$ $\times 10^{-3}, 9.7 \times 10^{-3}, 9.2 \times 10^{-3}, 9.0 \times 10^{-3}$, and $8.1 \times 10^{-3}$ where the value for $N_{B}=5$ deviates most from a linear correlation. The mean square errors of $H / N_{B}$ with respect to a linear correlation are practically the same for the unconstrained and the constrained results with a marginal advantage for the constrained results.

If we consider now the values for $n_{B b}$ in Table 3 we observe much larger discrepancies between the unconstrained and the constrained results with the largest discrepancy occurring for $N_{B}=1$ which was already mentioned above. Looking on Table 3 we see that for the unconstrained simulations the solute bulk fluid density for 1:399 is even slightly higher than for 2:398, which is rather striking. If we form the ratios $n_{B b} / N_{B}$, we obtain in the series $N_{B}=1,2,5,10,20,30,40$ for the unconstrained results the values $7.5 \times 10^{-4}, 3.6 \times 10^{-}$ 
${ }^{4}, 4.0 \times 10^{-4}, 4.3 \times 10^{-4}, 3.2 \times 10^{-4}, 3.8 \times 10^{-4}$, and $3.8 \times 10^{-4}$, and for the constrained results the values $2.1 \times 10^{-4}, 3.0 \times 10^{-4}, 2.1 \times 10^{-4}, 3.1 \times 10^{-4}, 3.2 \times 10^{-4}, 3.0 \times 10^{-4}$, and $3.2 \times 10^{-4}$. Looking on the unconstrained values, we observe an extreme value for $N_{B}=1$, whilst the other results for $n_{B b} / N_{B}$ lie between $3.2 \times 10^{-4}$ and $4.3 \times 10^{-4}$. Considering the constrained $n_{B b} / N_{B}$ values, the results for $N_{B}=1$ and 5 are $2.1 \times 10^{-4}$ and all the other values lie between 3.0 $\mathrm{x} 10^{-4}$ and between $3.2 \times 10^{-4}$. We note that all unconstrained values are higher than the constrained values for which we do not have an explanation. But we observe the satisfying tendency that the relative deviations become smaller with increasing $N_{B}$. Moreover, we see that the $n_{B b} / N_{B}$ values from the constrained simulations show less scattering than those from the unconstrained simulations.

Next we consider the adsorption excess values $\Gamma$ given in Table 3 . If we form again the ratios $\Gamma / N_{B}$, we obtain in the series $N_{B}=1,2,5,10,20,30,40$ for the unconstrained results the values $1.9 \times 10^{-3}, 3.4 \times 10^{-3}, 3.2 \times 10^{-3}, 3.1 \times 10^{-3}, 3.5 \times 10^{-3}, 3.2 \times 10^{-3}$, and $3.1 \times 10^{-3}$, and for the constrained results the values $4.1 \times 10^{-3}, 3.7 \times 10^{-3}, 4.1 \times 10^{-3}, 3.6 \times 10^{-3}, 3.5 \times 10^{-3}, 3.5$ $\mathrm{x} 10^{-3}$, and 3.3.x10 $0^{-3}$. The fact that the results from the unconstrained simulations are always smaller than those from the constrained simulations is simply a consequence of the fact that the bulk fluid densities of the unconstrained simulations are higher than those of the constrained simulations.

Let us now approach the adsorption isotherm. In Fig. 7 the adsorption excess values $\Gamma$ as function of the bulk solute densities $n_{B b}$ from the unconstrained and constrained simulations given in Table 3 are shown in graphical form. For a clearer presentation the high dilution results are enlarged in the inset. For the particle ratio 40:360 the results from both unconstrained simulation runs are shown separately together with the averaged value. 
For a further discussion of the adsorption data we first consider the Henry constant. For the constrained simulations we obtain directly from the entrances for $N_{B}=1$ in Table 3 the value $H_{d i r, c o n} *=19.48$ which is identical with the value from Eq. (17). We remind that the Henry constant yields the slope of the tangent to the adsorption isotherm in the limit of infinite dilution. This tangent is also shown in Fig. 7. For the unconstrained simulations a direct calculation of the Henry constant does not make much sense in view of the inconsistency of the results for 1:399 and 2:398.

Next we looked for a correlation of the adsorption isotherm $\Gamma=\Gamma\left(n_{B b}\right)$ by the Langmuir equation [21]

$$
\Gamma_{c o r r} *=a n_{B b} /\left(1+b n_{B b}\right)
$$

The fit using the method of least squares gave for the constrained simulation results the parameters $a_{c o n}=13.16$ and $b_{c o n}=21.18$ with a mean square error $(\mathrm{MSE})=1.4 \times 10^{-5}$ and in the unconstrained case the parameters $a_{u n}=9.69$ and $b_{u n}=12.08$ with MSE $=3.3 \times 10^{-5}$. The resulting Langmuir isotherms are also shown in Fig. 7 and we see that they fit the data reasonably good. Considering the value of the MSE as some measure for the scattering of the simulation results, the constrained simulations show again less scattering than the unconstrained simulations.

\subsection{Orientation and Selectivity}

Besides the local density of the CoM it is also of importance to give statements about the most preferred orientation of an ethane molecule in the simulation system. Figure 8 shows the orientation parameter $P(z)[22]$, which is defined as

$$
P(z)=0.5(3<\cos 2 \theta>-1)
$$


where $\theta$ indicates the angle between the adsorbing wall and the molecule axes of ethane. The ethane molecule is parallel to the wall for $\theta=0^{\circ}$, and perpendicular to the wall for $\theta=$ $90^{\circ}$. Simulations have been performed for a 1:399 ethane-methane system according to the abovementioned specifications for constrained simulations. The angular brackets <...> denote averaging over the orientations of ethane at fixed positions of the CoM. If the ethane molecule is parallel to the wall $<\cos ^{2} \theta>$ equals 1 and thus the order parameter $P(z)=1$. If ethane is orientated isotropically, then $<\cos ^{2} \theta>$ becomes $1 / 3$ and $P(z)=0$. Furthermore, for the perpendicular case $<\cos ^{2} \theta>$ becomes 0 and the order parameter $P(z)=-1 / 2$. Simulation results for the order parameter $P(z)$ as function of the distance $z$ from the wall are shown in Fig. 7 as well as the local density profile for the corresponding ethane molecule.

Apparently close to the wall ethane is preferably parallel adsorbed. Similarly the orientation of the ethane molecule in the second and the third adsorption peaks is rather parallel. In the valleys between the density peaks ethane is orientated somewhat perpendicularly. With increasing distance to the wall the orientation parameter $P(z)$ wobbles round 0 what signifies an isotropical orientation in the centre of the simulation box.

Fig. 9 shows results for the selectivity $S_{B A}$ as defined in Eq. (18) as measure for the separation of ethane from methane depending on the mole fraction of ethane in the bulk fluid achieved over the PMF. With decreasing mole fractions of ethane the selectivity $S_{B A}$ is rising according the ratio between the first density peak of ethane related to its bulk density. The presented results match in their order of magnitude with selectivity values of other adsorption systems for either plane walls [12] or slit pores [23]. Somewhat higher values of $S_{B A}$ are caused by lower system temperatures [24]. 


\section{Summary and Conclusion}

Two methods have been applied in order to study the adsorption of a dilute component from a mixture on a plane wall by molecular dynamics simulations. Actually the model mimics the solution of ethane in methane as solvent close to a graphite surface. The one method is standard molecular dynamics which was called the unconstrained method. As alternative the mean force (MF) method was used where the mean force on a constrained solute molecule is integrated over a path from the bulk fluid to the wall. Here, an extension of this method was introduced, where the absolute value of the bulk density is determined by particle balance which makes it possible to calculate adsorption isotherms.

Before we arrive at a final conclusion, a broader background shall be displayed. We were confronted with the strong scattering of standard simulation results for the adsorption from dilute solutions for the case of spherical molecules [1]. This scattering was such strong that it did not allow the calculation of reliable density profiles within rather long runs up to several million time steps. For these systems, however, we obtained reasonable density profiles with the MF-method. As the unconstrained simulation results for the solutions of spherical molecules considered were much worse than in the present study the question arises, when the unconstrained simulations fail and hence constrained simulations should be performed. In our opinion standard simulations will yield bad results for two rather different situations a) for a very dilute solution if there is not a pronounced minimum in the potential of mean force, and b) in case if there is a very strong barrier in the potential of mean force. In case a) the very few solute particles stray around and hence give bad statistics as it happens for the spherical molecules considered in [1], and in case b) the 
solute particles may not be able to cross the barrier. The present 'simple' system was chosen such that it neither falls in the one or the other category in order to allow a comparison of the MF with the standard simulations.

Next, we want to emphasise that neither the results from the one or the other method are right or wrong. Definitely, the results from both simulation methods considered as well as from any other molecular simulation method suffer from statistical uncertainties. The question can only be which method shows less statistical uncertainties. In order to clarify that point we have performed several consistency checks which show that the results from constrained simulations show less scattering than those from the unconstrained simulations. We also found that in general the relative deviations between the results from both methods are larger for smaller concentrations of solute particles and decrease with increasing concentration.

Finally, some additional considerations on both methods might be appropriate. If we consider one solute particle in the constrained method this is fixed and feels only the force from the solvent particles. As there are many of them in the pore this should yield good statistics. This obviously is an advantage over the unconstrained simulations in which this solute molecule travels around in the system and causes larger statistical fluctuations. This statement is supported by our previous simulations for solutions of spherical molecules [1] as well as by the present work where the results from the unconstrained simulations for one solute molecule show strong inconsistencies. Let us now consider a larger number of solute particles, i.e. in our case $N_{B}$ values ranging from 10 to 40 . One would expect that the statistics become better for both methods which is actually confirmed by our results. We 
observe, however, also that the scattering of all considered quantities in this concentration range is less for the results from the constrained simulations than for the results from the unconstrained simulations. Finally, we want to discuss the constrained method in the intermediate concentration range, i.e. for the $N_{B}$ values 2 and 5 . In this case we have only a few freely moving solute particles in the system which could lead to some scattering in their mean force on the reference solute particle. From our analysis we observe that the reduced value $n_{B b} / N_{B}$ for $N_{B}=2$ fits well to the results for $N_{B}$ from 10 to 40 , whilst the reduced value $n_{B b} / N_{B}$ for $N_{B}=5$ fits to the result for $N_{B}=1$ which we do not really understand.

Summarising, we conclude that the MF method is superior over the standard method for infinite dilution but also for finite concentrations with a sufficiently large number of solute particles.

\section{Acknowledgements}

Financial support by Deutsche Forschungsgemeinschaft for the project 'Adsorption aus wässrigen Lösungen', Az Fi 287/13-2, within the priority research programme 'Molekulare Modellierung und Simulation in der Verfahrenstechnik' is gratefully acknowledged (R.T. and L.S.). The MACSIMUS development has been supported by the The Ministry of Education, Youth and Sports of the Czech Republic under the project LC512 (Center for Biomolecules and Complex Molecular Systems). Moreover, we thank the John von Neumann Institute für Computing, Jülich, Germany, for allocation of computer time at JUMP, project ID hvi020. 


\section{References}

[1] W. Billes, F. Bazant-Hegemark, M. Mecke, M. Wendland, J. Fischer. Change of Free Energy during Adsorption of a Molecule. Langmuir 19, 10862 (2003).

[2] W. Billes, R. Tscheliessnig, J. Fischer. Molecular simulation of adsorption from dilute solutions. Acta Biochim. Pol. 52, 685 (2005).

[3] R. Tscheliessnig, W. Billes, M. Wendland, J. Fischer, J. Kolafa. Adsorption of benzene from an aqueous solution. Mol. Sim. 31, 661 (2005).

[4] J. Kolafa. 'http://www.vscht.cz/fch/software/macsimus'

[5] S. Sokolowski, J. Fischer. Lennard-Jones mixtures in slit-like pores: a comparison of simulation and density-functional theory. Molec. Phys. 71, 393 (1990).

[6] R.F. Crachnell, D. Nicholson, N. Quirke. In Molecular Simulation and Industrial Applications, K.E. Gubbins, N. Quirke (Eds.), p. 459, Gordon and Breach, London (1997).

[7] J.-H. Yun, T. Düren, F.J. Keil, N. Saeton. Adsorption of Methane, Ethane, and Their Binary Mixtures on MCM-41: Experimental Evaluation of Methods for the Prediction of Adsorption Equilibrium. Langmuir 18, 2693 (2002).

[8] T. Voung, P.A. Monson. Monte Carlo Simulations of Adsorbed Solutions in Heterogeneous Porous Materials. Adsorption 5, 295 (1999).

[9] A. Vishnyakov, E.M. Piotrovskaya, E.N. Brodskaya. Liquid-Vapor Equilibrium and the Molecular Structure of Methane-Ethane Mixtures Adsorbed in a Mesopore. Russ. J. Phys. Chem. 74, 1500 (2000).

[10] D. Möller, J. Oprzynski, A. Müller, J. Fischer. Prediction of thermodynamic properties of fluid mixtures by molecular dynamics simulations: methane-ethane. Molec. Phys. 75, 363 (1992). 
[11] D.M. Ruthven. Priciples of Adsorption and Adsorption Processes, Wiley Interscience, New York (1982).

[12] M. Wendland, U. Heinbuch, J. Fischer. Adsorption of simple gas mixtures on a plane wall: Born-Green-Yvon results for structure, adsorption isotherm and selectivity. Fluid Phase Equil. 48, 259 (1989).

[13] A.D. MacKerell Jr., B. Brooks, C.L. Brooks III, L. Nilsson, B. Roux, Y. Won, M. Karplus. In The Encyclopedia of Computational Chemistry 1, P.v.R. Schleyer, et al. (Eds.), vol. 1, pp. 271-277, John Wiley, Chichester (1998).

[14] J.D. van der Waals. Molecular theory of a substance composed of two different Species (in Dutch). Z. Phys. Chem. 5, 133 (1889).

[15] H.A. Lorentz. Ueber die Anwendung des Satzes vom Virial in der kinetischen Theorie der Gase. Ann. Physik 12, 127 (1881).

[16] D. Berthelot. Sur le Mélange des Gaz. Compt. Rend. 126, 1703 and 1857 (1898).

[17] W.A. Steele. The interaction of gases with solid surfaces, Pergamon Press, Oxford (1974).

[18] A. Müller, J. Winkelmann, J. Fischer. Backone family of equations of state .1. Nonpolar and polar pure fluids AIChE-J. 42, 1116 (1996).

[19] D.D. Do, H.D. Do. Evaluation of 1-Site and 5-Site Models of Methane on Its Adsorption on Graphite and in Grahitic Slit Pores. J. Phys. Chem. B 109, 19288 (2005).

[20] U. Heinbuch, J. Fischer. On the application of Widom's test particle method to homogeneous and inhomogeneous fluids. Mol. Sim. 1, 109 (1987).

[21] I. Langmuir. Constitution and Fundamental Properties of Solids and Liquids. I. Solids. J. Am. Chem. Soc. 38, 2221 (1916). 
[22] J.G. Harris. Liquid-vapor interfaces of alkane oligomers: structure and thermodynamics from molecular dynamics simulations of chemically realistic models. $J$. Phys. Chem. 96, 5077 (1992).

[23] S. Wang, Y. Yu, G. Gao. Grand canonical Monte Carlo and non-equilibrium molecular dynamics simulation study on the selective adsorption and fluxes of oxygen/nitrogen gas mixtures through carbon membranes. J. Membr. Sci. 271, 140 (2006).

[24] J. Fox. Adsorption of hydrocarbons in porous materials: a computational study. PhD thesis, University of Edinburgh (2005). 


\begin{tabular}{|c|c|c|c|c|c|}
\hline Molecule & Bond & Bond length & Site & Site-site energy & Site-site diameter \\
\hline & & $l[\mathrm{~nm}]$ & & $\varepsilon_{\alpha d} / k[\mathrm{~K}]$ & $\sigma_{\alpha \alpha}[\mathrm{nm}]$ \\
\hline $\mathrm{CH}_{4}$ & $\mathrm{C}-\mathrm{H}$ & 0.10900 & & & \\
\hline $\mathrm{CH}_{4}$ & & & $\mathrm{C}$ & 45.47 & 0.3207 \\
\hline $\mathrm{CH}_{4}$ & & & $\mathrm{H}$ & 21.14 & 0.2370 \\
\hline $\mathrm{C}_{2} \mathrm{H}_{6}$ & $\mathrm{CH}_{3}-\mathrm{CH}_{3}$ & 0.1540 & & & \\
\hline $\mathrm{C}_{2} \mathrm{H}_{6}$ & & & $\mathrm{CH}_{3}$ & 91.19 & 0.3858 \\
\hline $\mathrm{C}$ & & & $\mathrm{C}$ & 25.18 & 0.3635 \\
\hline
\end{tabular}


Table 2

Pore widths $L$ and initial positions of the solute particles. For the unconstrained simulations two runs over 300,000 time steps were made with initial positions for the $N_{B}$ solutes as given. For the constrained simulations 60 runs over 10,000 time steps were made with always the same initial positions for the $N_{B}-1$ freely moving solutes and the constrained particle at the prescribed distance from the wall. $\mathrm{C}$ means centre of the pore, L left half, $\mathrm{R}$ right half. Up to $N_{B}=30, \mathrm{~L}$ and $\mathrm{R}$ also indicate insertion into the first layer, for $N_{B}=40$ the meaning of L1 and L2 is first and second layer.

\begin{tabular}{|l|l|l|l|l|}
\hline$N_{B}$ & $\begin{array}{l}\text { Pore width } L \\
{[\mathrm{~nm}]}\end{array}$ & $\begin{array}{l}\text { Unconstrained } \\
\text { Run 1 }\end{array}$ & $\begin{array}{l}\text { Unconstrained } \\
\text { Run 2 }\end{array}$ & Constrained \\
\hline 1 & 3.2196 & $1 \mathrm{C}$ & $1 \mathrm{~L}$ & $1 \mathrm{~L}, 1 \mathrm{R}$ \\
\hline 2 & 3.2219 & $1 \mathrm{C}, 1 \mathrm{~L}$ & $3 \mathrm{~L}, 2 \mathrm{R}$ & $1 \mathrm{C}$ \\
\hline 5 & 3.2289 & $1 \mathrm{C}, 2 \mathrm{~L}, 2 \mathrm{R}$ & $5 \mathrm{~L}, 5 \mathrm{R}$ & $2 \mathrm{~L}, 2 \mathrm{R}$ \\
\hline 10 & 3.2405 & $2 \mathrm{C}, 4 \mathrm{~L}, 4 \mathrm{R}$ & all random & $1 \mathrm{C}, 4 \mathrm{~L}, 4 \mathrm{R}$ \\
\hline 20 & 3.2634 & $2 \mathrm{C}, 9 \mathrm{~L}, 9 \mathrm{R}, 9 \mathrm{R}$ \\
\hline 30 & 3.2861 & $4 \mathrm{C}, 13 \mathrm{~L}, 13 \mathrm{R}$ & all random & $3 \mathrm{C}, 13 \mathrm{~L}, 13 \mathrm{R}$ \\
\hline 40 & 3.3084 & $4 \mathrm{C}, 13 \mathrm{~L} 1,5 \mathrm{~L} 2$, & all random & $3 \mathrm{C}, 13 \mathrm{~L} 1,5 \mathrm{~L} 2,13 \mathrm{R} 1$, \\
& & $13 \mathrm{R} 1,5 \mathrm{R} 2$ & & $5 \mathrm{R} 2$ \\
\hline
\end{tabular}


Table 3:

Reduced bulk densities $n_{B b}{ }^{*}$ and adsorption excess values $\Gamma^{*}$ for the unconstrained and the constrained case for $N_{B}=1,2,5,10,20,30$, and 40 .

\begin{tabular}{|l|l|l|l|l|}
\hline & \multicolumn{2}{|c|}{ Unconstrained } & \multicolumn{2}{c|}{ Constrained } \\
\hline$N_{B}$ & $n_{B b^{*}}$ & $\Gamma^{*}$ & $n_{B b^{*}}$ & $\Gamma^{*}$ \\
\hline 1 & $7.51 \times 10^{-4}$ & $1.86 \times 10^{-3}$ & $2.10 \times 10^{-4}$ & $4.09 \times 10^{-3}$ \\
\hline 2 & $7.28 \times 10^{-4}$ & $6.89 \times 10^{-3}$ & $6.04 \times 10^{-4}$ & $7.39 \times 10^{-3}$ \\
\hline 5 & $20.07 \times 10^{-4}$ & $16.30 \times 10^{-3}$ & $10.38 \times 10^{-4}$ & $20.33 \times 10^{-3}$ \\
\hline 10 & $42.67 \times 10^{-4}$ & $31.14 \times 10^{-3}$ & $30.88 \times 10^{-4}$ & $36.19 \times 10^{-3}$ \\
\hline 20 & $64.81 \times 10^{-4}$ & $69.27 \times 10^{-3}$ & $64.01 \times 10^{-4}$ & $69.62 \times 10^{-3}$ \\
\hline 30 & $113.42 \times 10^{-4}$ & $94.68 \times 10^{-3}$ & $90.3 \times 10^{-4}$ & $104.4 \times 10^{-3}$ \\
\hline 40 & $150.89 \times 10^{-4}$ & $123.3 \times 10^{-3}$ & $129.3 \times 10^{-4}$ & $132.4 \times 10^{-3}$ \\
\hline
\end{tabular}




\section{Figure captions}

FIG. 1: Local density profiles $n_{B}(z)$ of ethane in dilute liquid solutions with methane from 2 unconstrained simulations with different inset positions of ethane for an ethane-methane ratio of 1:399 after 300,000 simulation steps each: - run $1, \cdots$ run 2 . The density profile of pure methane $n_{A}(z)$ is indicated by $(-\cdot-)$.

FIG. 2: Mean force $(. \cdot$ o.$\cdot)$ and potential of mean force (-) of a constrained ethane molecule in a mixture with 399 methanes as function of the normal distance $z$ from the 9/3 LJ wall after 10,000 time steps per simulation point. Grey areas indicate a repelled ethane molecule, white areas an ethane molecule attracted to the wall.

FIG. 3: Local density profiles $n_{B}(z)$ of ethane diluted in methane at two different ethanemethane ratios for the unconstrained and the constrained case. (-) 1:399 unconstrained, (....) 2:398 unconstrained after 600,000 simulation steps each, (- -) 1:399 constrained, and $(-\cdot-)$ 2:398 constrained after 10,000 time steps per simulation point.

FIG. 4: Local density profiles $n_{B}(z)$ of ethane diluted in methane at two different ethanemethane ratios for the unconstrained and the constrained case. (-) 5:395 unconstrained, (…) 10:390 unconstrained after 600,000 simulation steps each, (- -) 5:395 constrained, and $(-\cdot-)$ 10:390 constrained after 10,000 time steps per simulation point.

FIG. 5: Local density profiles $n_{B}(z)$ of ethane diluted in methane at two different ethanemethane ratios for the unconstrained and the constrained case. (-) 20:380 unconstrained, (…) 30:370 unconstrained after 600,000 simulation steps each, (- -) 20:380 constrained, and $(-\cdot-)$ 30:370 constrained after 10,000 time steps per simulation point.

FIG. 6: Local density profiles $n_{B}(z)$ of ethane in dilute liquid solutions with methane for an ethane-methane ratio of 40:360 from 2 unconstrained simulations with different inset positions of ethane after 300,000 simulation steps each: (-) run 1 and ( $\cdots$ ) run 2, and for the constrained case $(-\cdot-)$ after 10,000 time steps per simulation point.

FIG. 7: Adsorption excess $\Gamma^{*}$ versus solute bulk density $n_{B b}{ }^{*}$ results for ethane from constrained $(\bullet)$ and unconstrained $(\mathrm{x})$ simulations over 600,000 time steps. $\boldsymbol{\Delta}$ results from 
two separate unconstrained runs over 300,000 time steps, (-) adsorption isotherm from constrained and (- - ) unconstrained simulations fitted by the Langmuir equation, (....) Henry tangent calculated via the PMF, Eq. (17).

FIG. 8: Orientational order parameter $P(z)(-)$ and the local density profile $n_{B}(z)$ (grey area) of an ethane molecule for a 1:399 mixture.

FIG. 9: Selectivity $S_{B A}(\bullet)$ for several ethane-methane mixtures depending on the density of ethane in the bulk fluid $n_{B b}{ }^{*}$. ( ...) indicates the resulting tendency of the selectivity. 


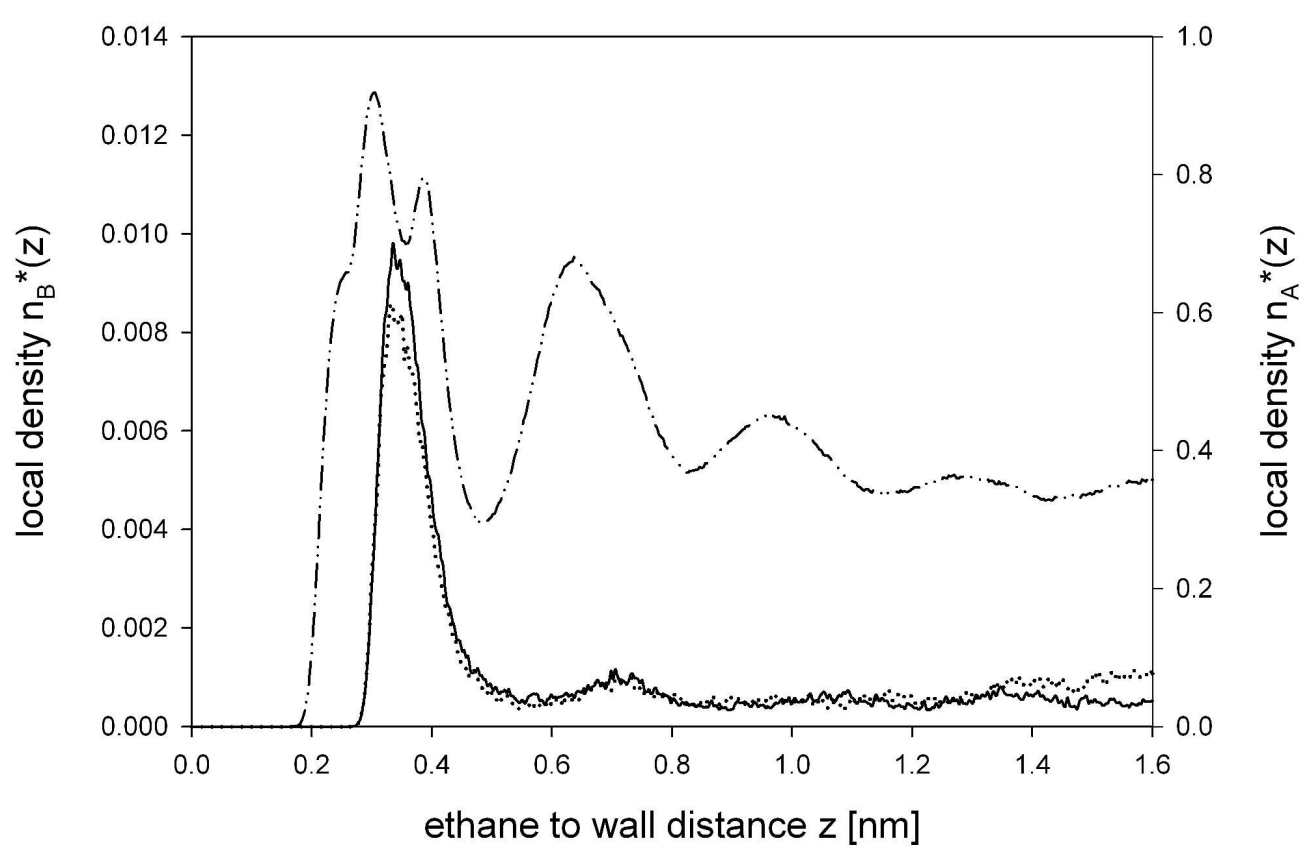

Figure 1

$679 \times 448 \mathrm{~mm}(150 \times 150 \mathrm{DPI})$ 


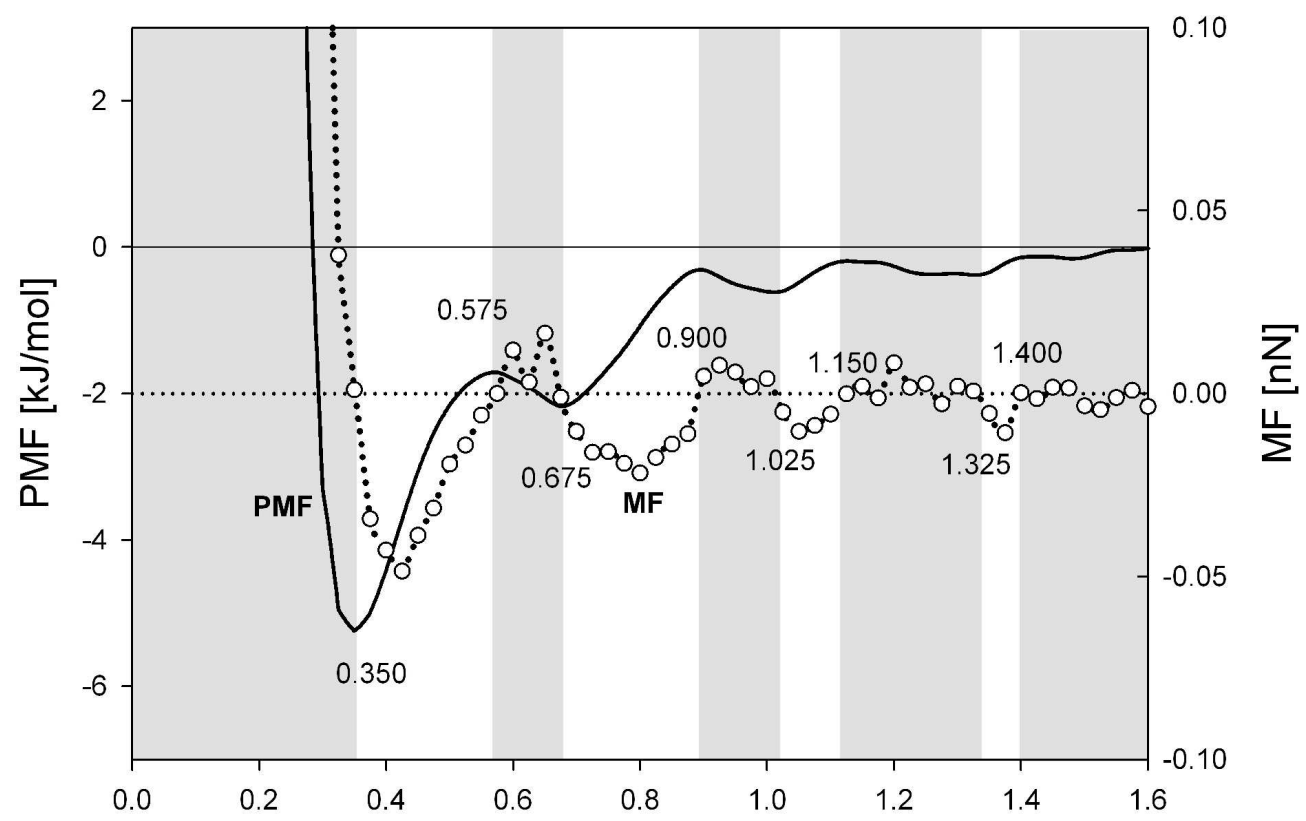

Ethane to wall distance $\mathrm{z}[\mathrm{nm}]$

Figure 2

$640 \times 443 \mathrm{~mm}(150 \times 150 \mathrm{DPI})$ 


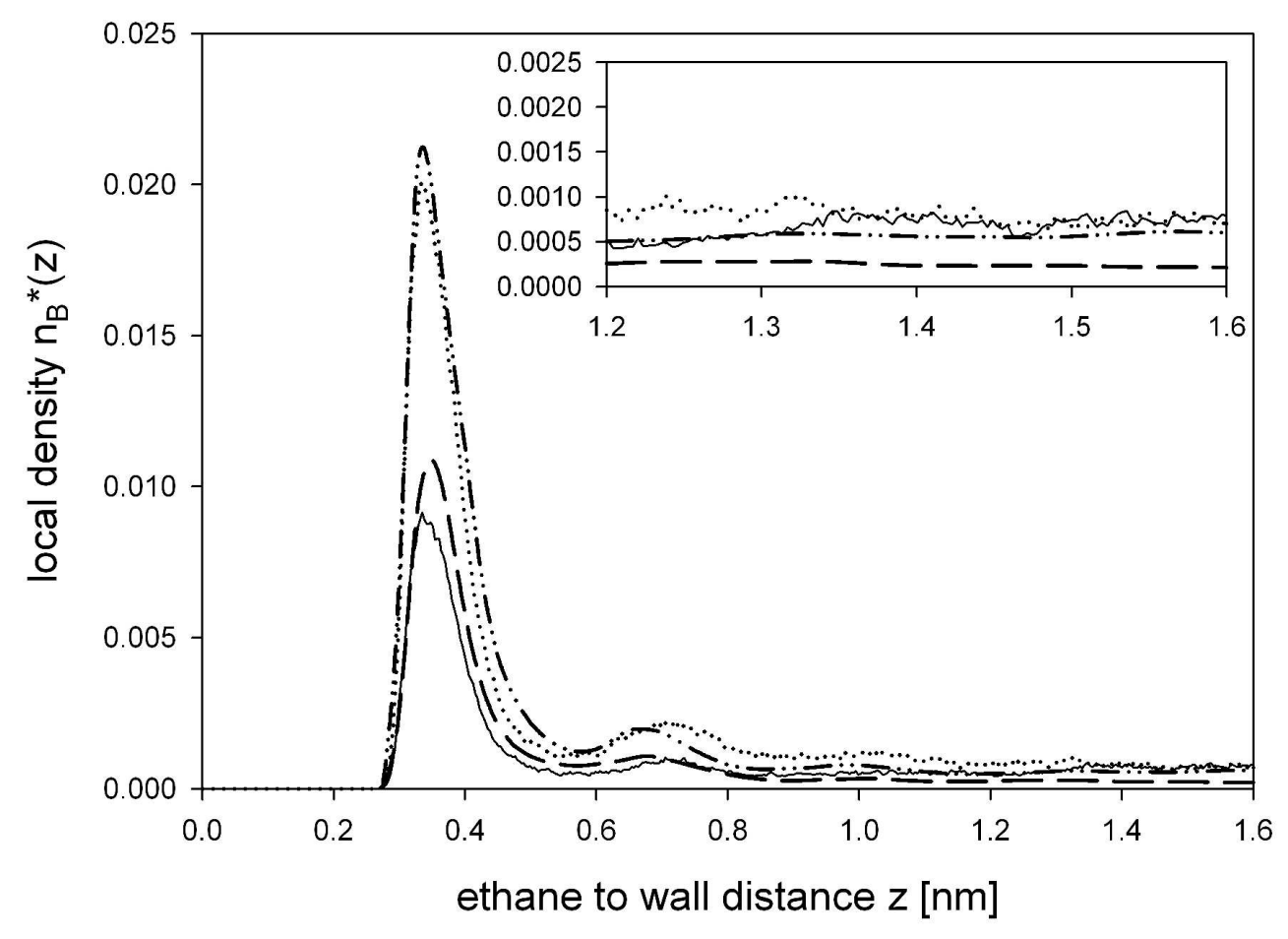

Figure 3

$609 \times 441 \mathrm{~mm}(150 \times 150 \mathrm{DPI})$ 


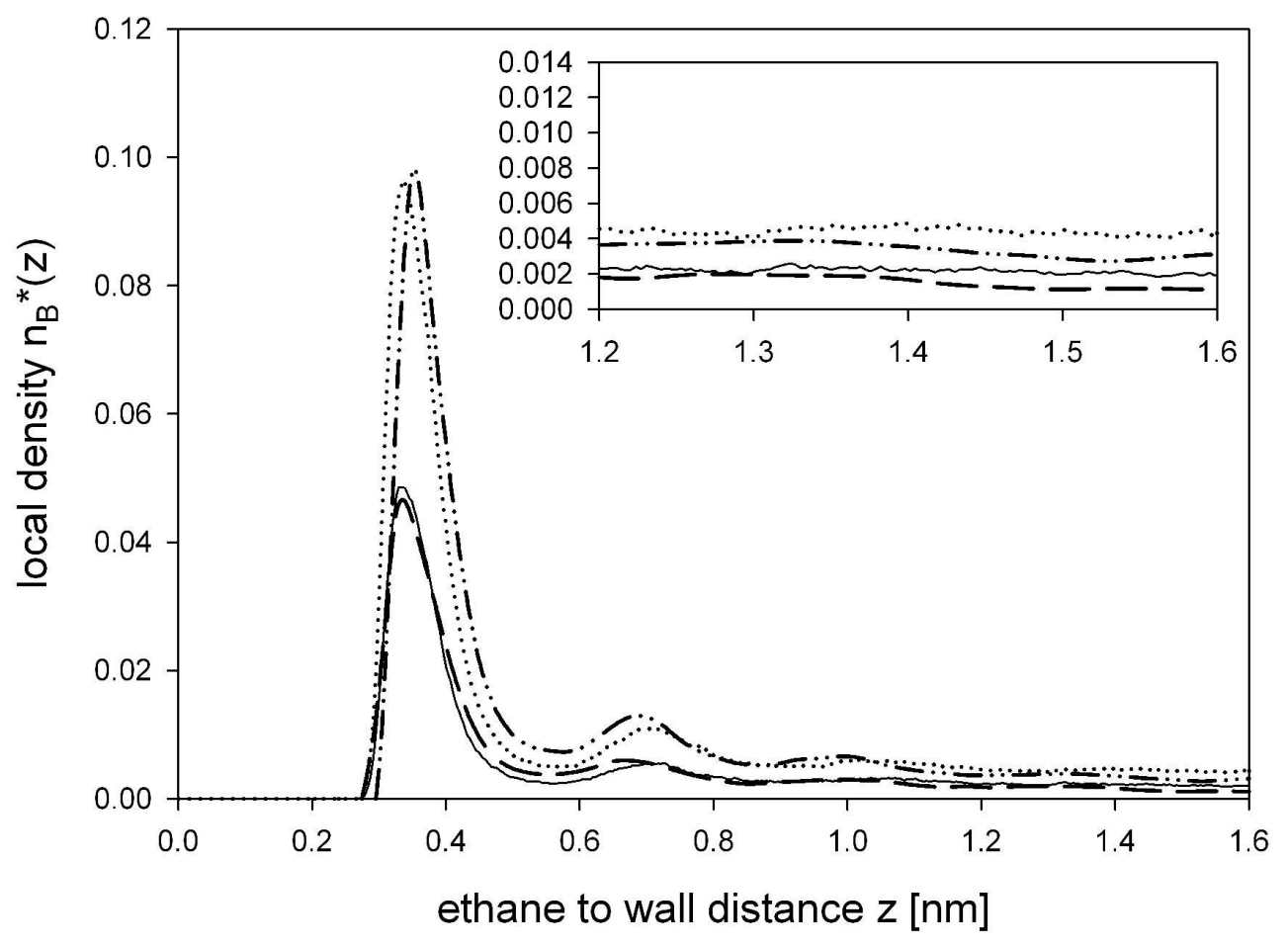

Figure 4 $601 \times 441 \mathrm{~mm}(150 \times 150 \mathrm{DPI})$ 


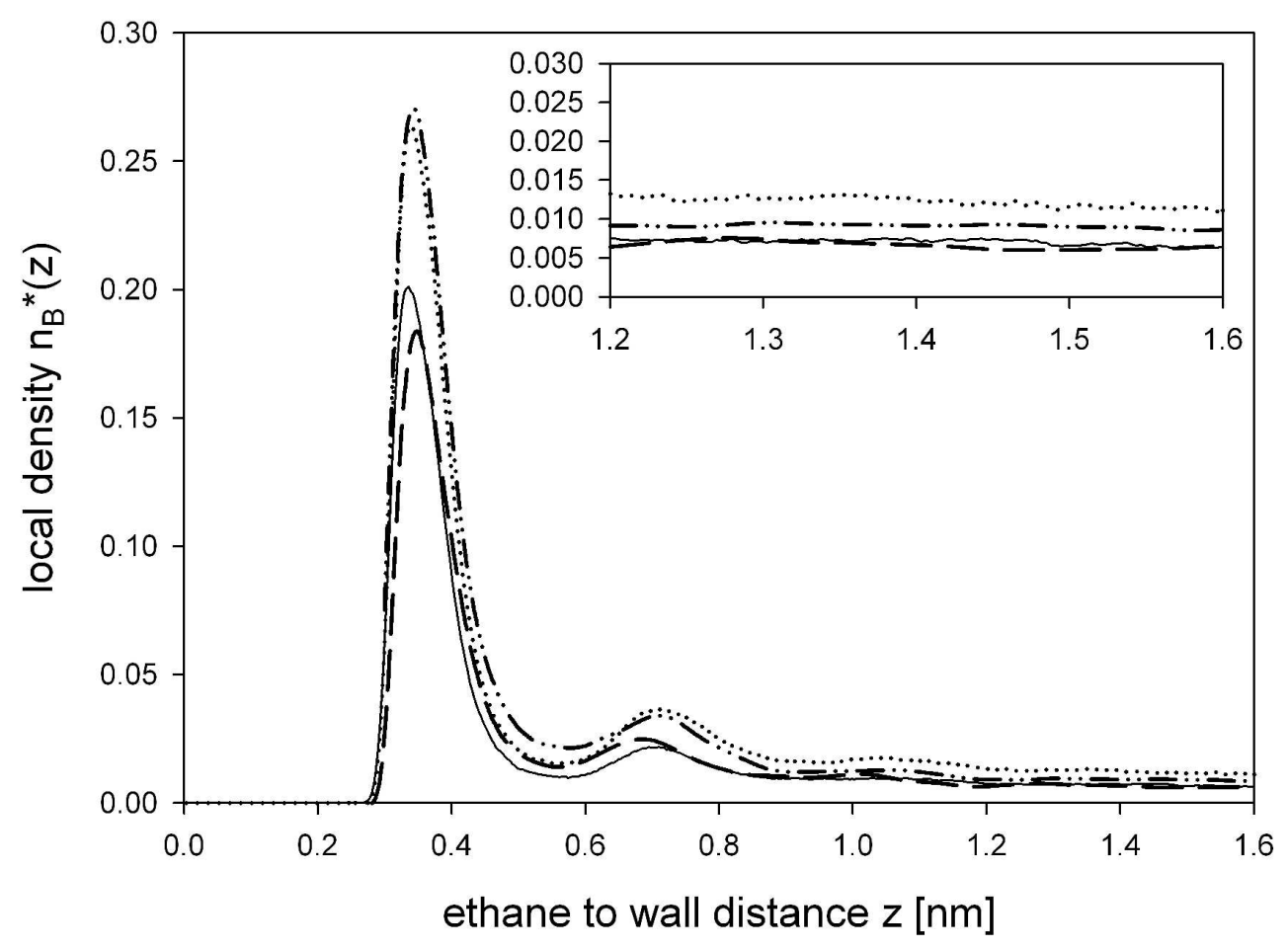

Figure 5

$601 \times 443 \mathrm{~mm}(150 \times 150$ DPI $)$ 


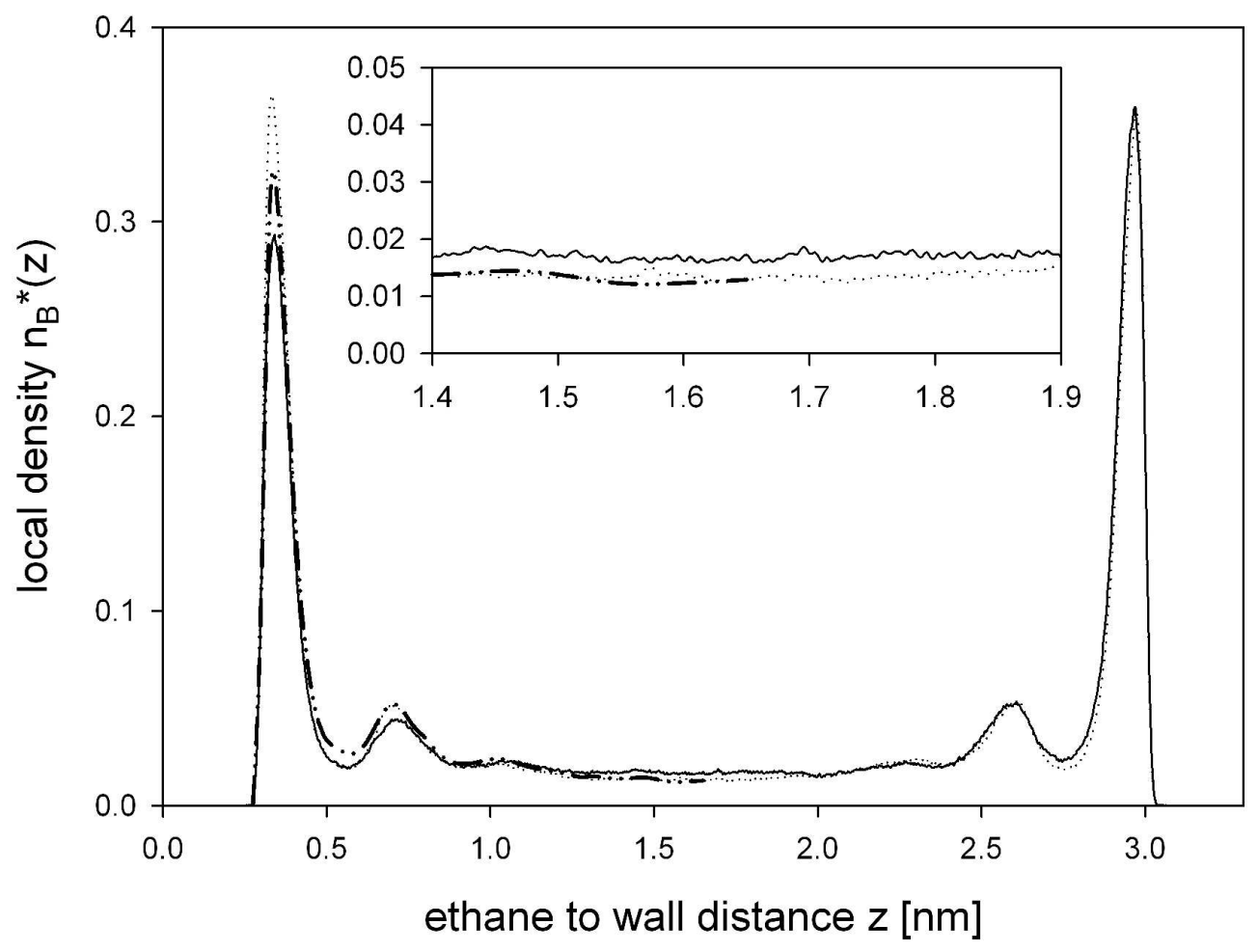

Figure 6

$581 \times 436 \mathrm{~mm}(150 \times 150$ DPI $)$ 


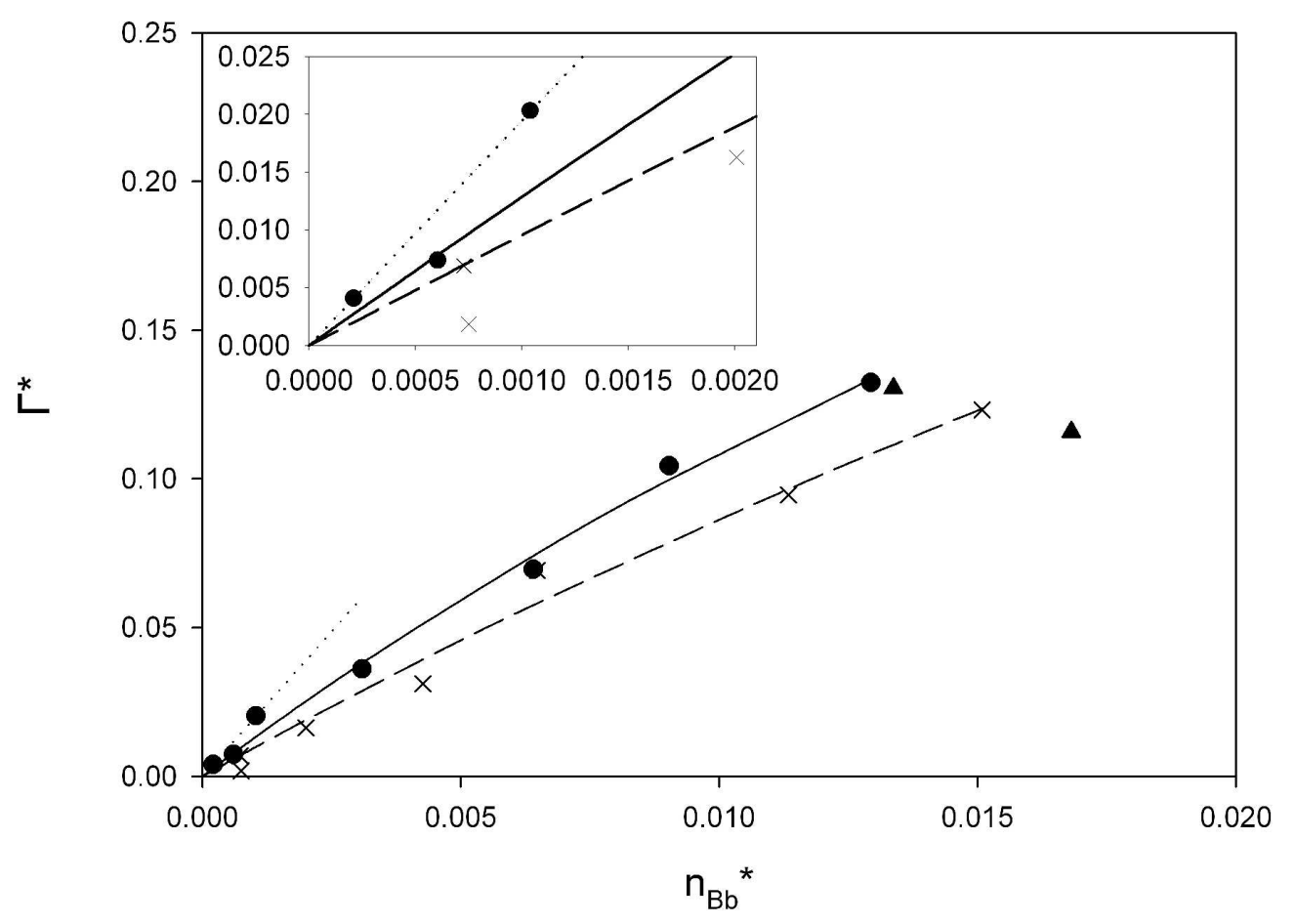

Figure 7

$615 \times 442 \mathrm{~mm}(150 \times 150 \mathrm{DPI})$ 


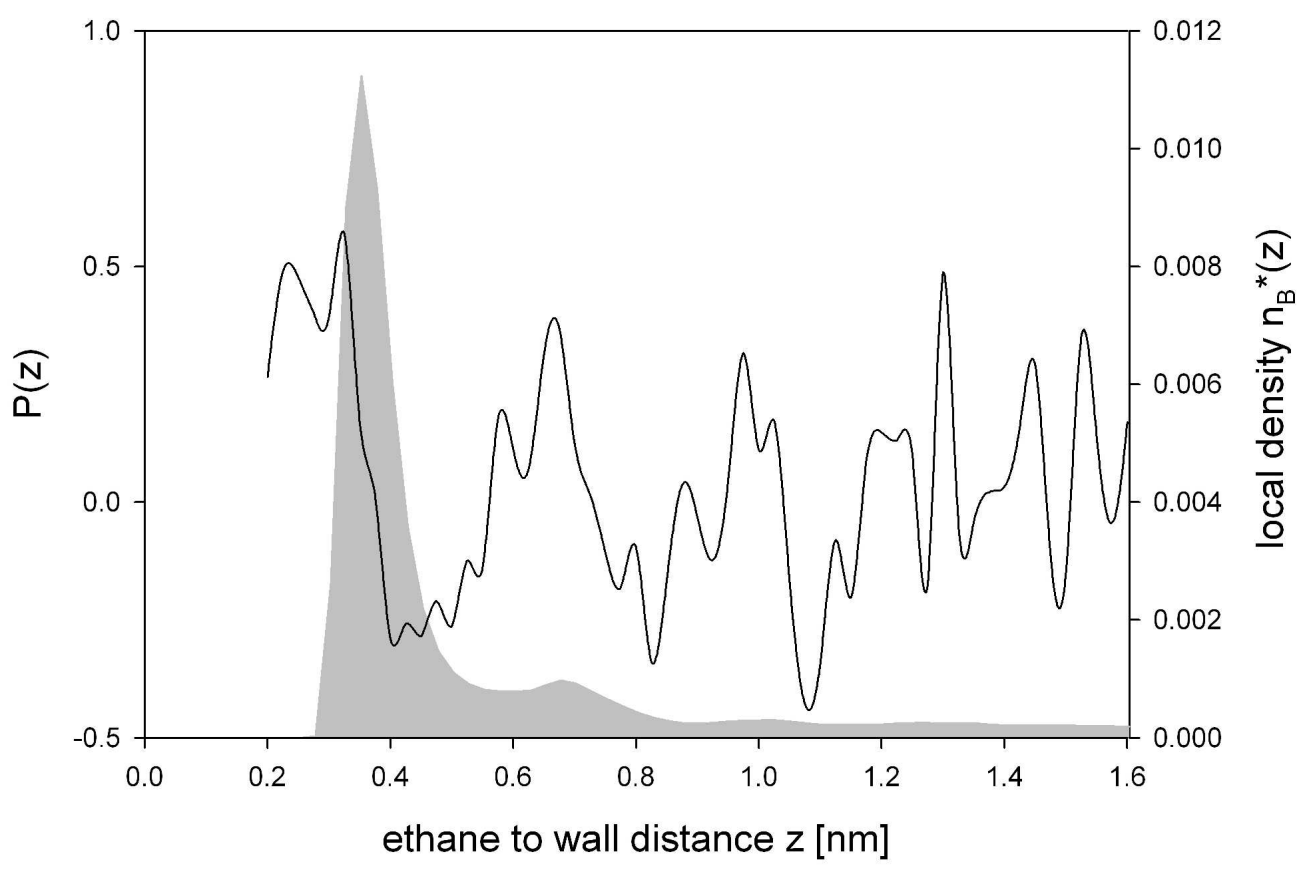

Figure 8 $662 \times 441 \mathrm{~mm}(150 \times 150 \mathrm{DPI})$ 


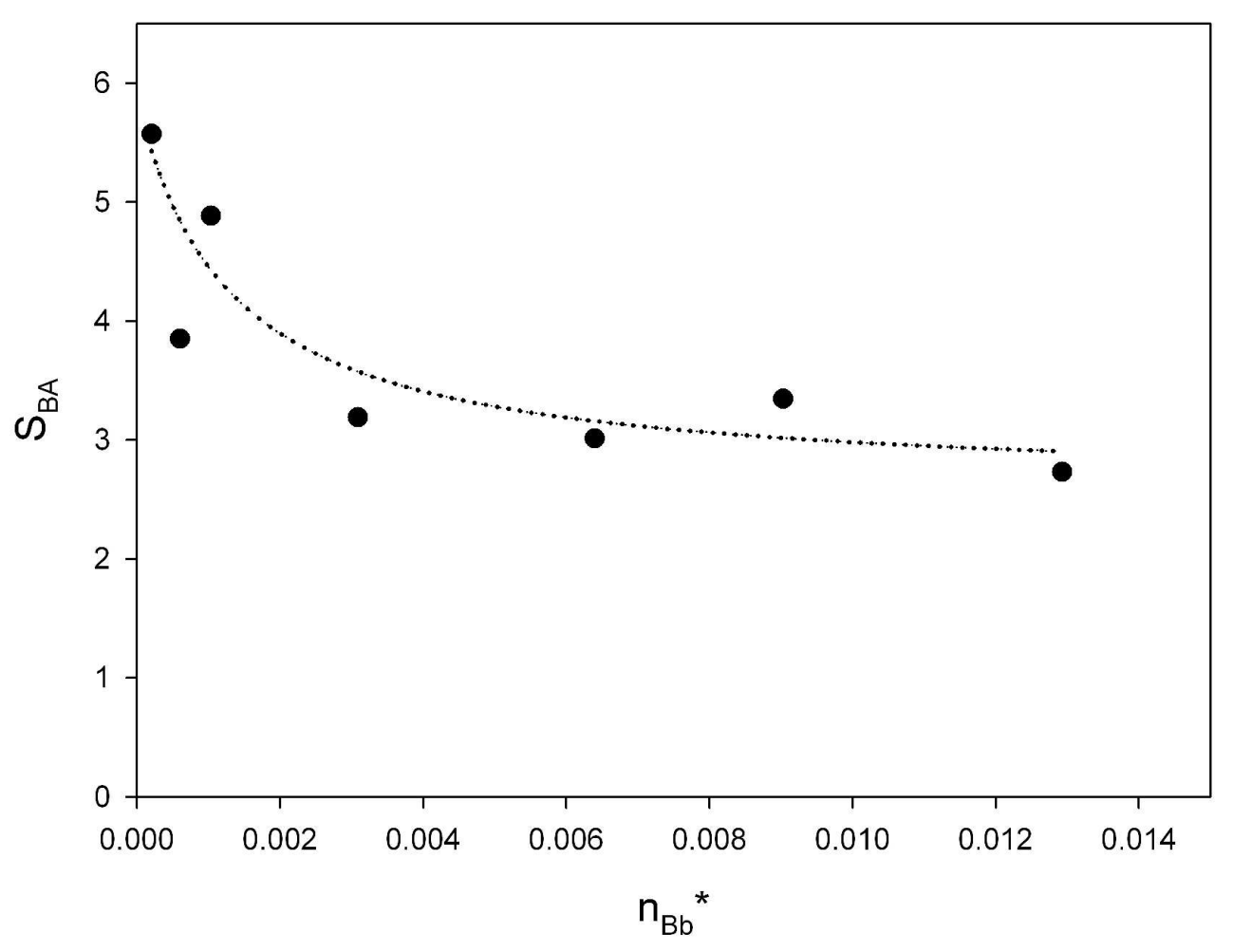

Figure 9

$575 \times 445 \mathrm{~mm}(150 \times 150 \mathrm{DPI})$

http://mc.manuscriptcentral.com/tandf/jenmol 